\title{
Personalidade e comportamentos de risco de motoristas: diferenças entre sexos
}

\author{
José Poças Correia' \\ Maria da Purificação Cunha Horta \\ Universidade de Lisboa, Lisboa - Portugal
}

\begin{abstract}
Resumo: $O$ traço de personalidade procura de sensações está associado a comportamentos de risco na condução, com implicações na vida em sociedade. Este estudo teve como objectivo verificar se o sexo diferencia o traço procura de sensações e a propensão para comportamentos de risco na condução. Foram avaliados I 40 motoristas (70 homens, 70 mulheres), com idades entre 25 e 55 anos. Como instrumentos, utilizaram-se um questionário sociodemográfico e as escalas SSS-V e DBQ. Verificou-se que os motoristas masculinos cometem mais infrações e apresentam escores mais elevados na procura de emoção e aventura (TAS), enquanto as mulheres apresentam maior propensão para a busca de experiências (ES) e uma correlação mais forte entre o traço procura de sensações e o cometimento de lapsos e infrações de trânsito. O sexo diferencia o traço procura de sensações e a sua associação aos comportamentos de risco na condução, devendo ser considerado na formação de futuros motoristas.
\end{abstract}

Palavras-chave: avaliação psicológica; personalidade; procura de sensações; sexo; motoristas.

\section{PERSONALITY AND RISKY BEHAVIOURS OF DRIVERS: SEX DIFFERENCES}

\begin{abstract}
The sensation seeking personality trait is associated with some risky driving behaviours, which has consequences in society. The aim of this study was to verify whether sex differentiates the sensation-seeking trait and the propensity for risky driving behaviours. 140 drivers were evaluated ( 70 men, 70 women), aged between 25 and 55 years. The instruments used were a demographic questionnaire and scales SSS-V and DBQ. It was found that male drivers commit more violations and have higher scores in thrill and adventure seeking (TAS), while women are more prone to seek experiences (ES) and a stronger correlation between the trait of sensation seeking and the commitment of lapses and traffic violations. Results suggested that sex differentiates sensationseeking trait and its association to risky driving behaviours, what should be considered in the training of new drivers.
\end{abstract}

Keywords: psychological assessment; personality; sensation seeking; sex; drivers.

\section{PERSONALIDAD Y CONDUCTAS DE RIESGO DE LOS CONDUCTORES: DIFERENCIAS DE SEXO}

Resumen: El rasgo de personalidad de búsqueda de sensaciones se asocia con comportamientos de riesgo en la conducción, teniendo implicaciones por la vida en la sociedad.

1 Endereço para correspondência: José Poças Correia, Faculdade de Medicina da Universidade de Lisboa, Instituto de Formação Avançada, Avenida Professor Egas Moniz, s/n, Lisboa - Portugal. CEP: 1649-028. E-mail: jpocascorreia @hotmail.com. 


\begin{abstract}
El objetivo de este estudio fue verificar si el sexo es diferente en el rasgo búsqueda de sensaciones y en la propensión a las conductas de riesgo en el tráfico. Se evaluaron 140 conductores ( 70 hombres, 70 mujeres), de entre 25 y 55 años. Los instrumentos utilizados fueron un cuestionario demográfico y escalas SSS-V y DBQ. Los hombres conductores cometen más infracciones de tráfico y tienen puntuaciones más altas en búsqueda de emoción y aventura (TAS), mientras que las mujeres tienen una mayor propensión para buscar experiencias (ES) y tienen una fuerte correlación entre el rasgo de búsqueda de sensaciones y la tendencia para los lapsos y las violaciones de tráfico. La diferencia de sexo en el rasgo búsqueda de sensaciones y su asociación con conductas de riesgo en el tráfico, debería tenerse en cuenta en la formación de los futuros conductores.
\end{abstract}

Palabras clave: evaluación psicológica; personalidad; búsqueda de sensaciones; sexo; conductores.

Os custos com os acidentes de trânsito podem representar $1 \%$ a 1,5\% do Produto Interno Bruto de cada país, tendo consequências que se manifestam em diferentes níveis da sociedade, desde a saúde física e psicológica de quem é envolvido em acidentes até à sensação de insegurança e impunidade de quem circula no trânsito. Os acidentes de trânsito, as infrações e a condução perigosa são um fenómeno social da actual vida moderna, sendo alvo de estudos que os tentam compreender, bem como prevenir. No Brasil e em muitos outros países, foi já instituída a obrigatoriedade da avaliação psicológica para adquirir a carteira de habilitação, tentando conhecer características individuais do motorista que possam predispor à ocorrência de acidentes ou protegê-lo dela. Numa recente revisão da literatura sobre estudos empíricos internacionais, Santos, Boff e Konflanz (2012) concluíram que, apesar de no caso brasileiro não existir consenso entre os profissionais da área sobre as características da personalidade a avaliar e os respectivos métodos psicométricos, isso não invalida a existência de inúmeros estudos internacionais que referem a necessidade de avaliar alguns traços de personalidade como a agressividade, a raiva na condução e procura de sensações, pois parecem predizer comportamentos de direção perigosa que potenciam a ocorrência de acidentes de trânsito e colocam em risco a vida em sociedade. Tal como referem Oliveira e Pinheiro (2007), o trânsito é vivido pelas pessoas como uma apropriação privada do espaço público, que se percebe pela forma como dirigem as suas viaturas e cometem todo o tipo de infrações, provocando estas frequentemente acidentes de trânsito com vítimas fatais. Apesar de a personalidade não ser o único elemento preditivo das infrações e dos acidentes de trânsito, Santos et al. $(2012$, p. 107) concluíram que "existem evidências científicas por detrás da necessidade de avaliação dos traços de personalidade em futuros motoristas. Tais características contribuem para os resultados das ocorrências de trânsito".

Considerando que o fator humano é a principal causa direta dos acidentes de trânsito, alguns autores têm identificado a raiva, o stresse, a ansiedade, a agressividade e a angústia como estados emocionais que mais afetam de forma negativa as capacidades de processamento informacional dos motoristas para conduzir em segurança. À semeIhança de outros investigadores, Bartholomeu (2008) identificou a busca por novas emoções e aventuras como um dos fatores da personalidade mais potenciadores do 
envolvimento em condutas de risco e violação das normas do trânsito. Este traço de personalidade tem suscitado muita atenção na investigação sobre o comportamento dos motoristas, havendo já países como a Austrália (NSW, 2011) onde as autoridades medem o índice de sensation seeking dos candidatos à obtenção da carteira de habilitação como fator de risco para os acidentes. Para Zuckerman (1994), a procura de sensações ou sensation seeking é um traço de personalidade que se revela através da procura de sensações e experiências variadas, novas, complexas e intensas e pela intenção de correr riscos físicos, sociais, legais e financeiros por causa das mesmas. Traduz-se numa tendência optimista para abordar novos estímulos e para explorar o ambiente, levando à aceitação do risco pelo prazer que isso acarreta.

Jonah (1997), num estudo de revisão da literatura, verificou que, dos 40 trabalhos analisados, apenas quatro não revelavam uma relação positiva entre a procura de sensações e alguns comportamentos de risco na condução, como a falta do uso do cinto de segurança, excesso de velocidade e variadas infrações de trânsito. Este traço parece influenciar não apenas a forma como os motoristas percepcionam o risco na condução e respondem a ele, mas também como se adaptam às mudanças originadas pela percepção desse risco. Essa adaptação está frequentemente relacionada com o aumento das condições de segurança ativa e passiva dos veículos, bem como com as condições da via em que se circula. Assim, a procura de sensações influenciaria a adaptação comportamental ao risco, levando a que os motoristas que procuram sensações tirem partido das melhores condições técnicas de segurança dos veículos, permitindo-lhes uma condução mais arriscada, enquanto os outros motoristas tenderiam a manter o mesmo comportamento e por conseguinte a beneficiar-se desse incremento de segurança adicional.

Algumas investigações têm permitido verificar que não existem diferenças significativas nos comportamentos de condução, aptidões e variáveis da personalidade, excepto para a procura de sensações, que permite prever as competências motoras e de segurança na origem da velocidade preferida dos motoristas. A procura de sensações tem sido também fortemente associada a vários tipos de infrações de trânsito, o que, segundo alguns autores, entre eles Jonah, Thiessen e Au-Yeung (2001), pode estar relacionado com excesso de velocidade, ultrapassagens frequentes e mudanças de faixa de rodagem, bem como condução sob fadiga e não utilização de cinto de segurança.

Alguns estudos (Fernandes, Hatfield, \& Job, 2010) sugerem que a relação entre a percepção do risco e a condução perigosa é de natureza diferente para homens e para mulheres. No entanto, relativamente ao sexo dos motoristas, há estudos que identificam diferenças de estilo e de comportamento de condução entre homens e mulheres (Reason, Manstead, Stradling, Baxter, \& Campbell, 1990; Constantinou, Panayiotou, Konstantinou, Loutsiou-Ladd, \& Kapardis, 2011). Relativamente às diferenças de sexo nos traços de personalidade que possam influenciar a condução, as mesmas são aferidas pelos estereótipos relacionados com as atitudes no trânsito. Para as mulheres, os comportamentos de poder e de imprudência representam a forma como o homem é percebido na sua relação social com o trânsito, ao passo que os homens consideram a 
mulher como sendo mais atenciosa e disciplinada, daí resultando uma condução mais prudente e cautelosa, o que nos pode levar a inferir que os homens são mais competitivos e as mulheres mais sociáveis no trânsito, exteriorizando um comportamento reflexo da sua história social e familiar. Outros estudos referem que os homens reportam mais acidentes e multas de trânsito (Lonczak, Neighbors, \& Donovan, 2007; Reason et al., 1990), constituindo um grupo de risco devido ao modo agressivo como conduzem e a características da personalidade que os levam a subestimar o perigo e a assumir mais riscos na condução, ao contrário das mulheres que, apesar de tenderem a ser mais stressadas do que os homens na condução, cometem menos infrações. De acordo com Jonah et al. (2001), os motoristas que procuram sensações adoptam um estilo de condução mais arriscado, embora não existam diferenças significativas quanto a idade, sexo, estatuto socioeconómico ou número de quilómetros percorridos. Em regra, os homens cometem mais infrações do que as mulheres (Reason et al., 1990), e os motoristas mais jovens têm um maior número de comportamentos de risco (Cooper, Pinili, \& Chen, 1994).

Os estudos mais recentes têm vindo claramente a identificar os motoristas masculinos, como aqueles que mais são autuados e os que reportam mais acidentes e com maior número de vítimas (Lonczak et al., 2007). Essas diferenças são tão significativas que, de acordo com a National Highway Traffic Safety Administration (2013), em 2002, a probabilidade de os motoristas americanos morrerem na estrada era três vezes superior nos homens do que nas mulheres. Para Lonczak et al. (2007), o sexo é um factor de diferenciação importante na condução, especialmente nas situações de trânsito que potenciam o aparecimento da raiva e os factores de risco como o consumo de álcool e a procura de sensações. Considerando que as mulheres são mais cuidadosas do que os homens na condução, isso poderá levá-las a irritarem-se mais quando observam comportamentos menos próprios nos outros motoristas, embora adoptem um conjunto de respostas mais adaptativas do que os homens (Jovanović, Stanojević, \& Stanojević, 2011). No que respeita à condução de risco, nomeadamente ao excesso de velocidade, existe uma maior pressão social nos homens do que nas mulheres, e é também neste tipo de comportamento de risco que o traço de personalidade procura de sensações é mais diferenciador para ambos os sexos (Cestac, Paran, \& Delhomme, 2011).

Apesar de os resultados obtidos em diversos estudos sugerirem que o género e o sexo dos motoristas influenciam de igual modo a expressão do seu estilo de condução, associando as características socioculturais da masculinidade (gênero) às competências do motorista experiente (being a skilful driver) e as características da feminilidade às competências do motorista seguro (being a safe driver) (Özkan \& Lajunen, 2006), é ao nível do sexo dos condutores, por ser de raiz biológica, que pretendemos aferir as principais diferenças comportamentais associadas à presença do traço procura de sensações. Com efeito, tal como referem Özkan e Lajunen (2006, p. 270), "O sexo tem uma conotação biológica e as diferenças entre sexos advêm das diferenças temperamentais inatas de cada sexo (como as teorias biológicas de Buss, 1995), ao passo que o gênero é sobretudo um conceito social e cultural". 
A prevalência de elevados níveis de sinistralidade rodoviária em muitos países e a evidência científica de que os acidentes de viação são maioritariamente devidos a factores humanos, bem como a existência de um número pouco significativo de estudos de língua portuguesa sobre esta temática, levaram-nos a tentar verificar se existem diferenças no traço procura de sensações entre motoristas de ambos os sexos e se este traço está relacionado com a propensão para comportamentos de risco na condução.

Tentamos que o estudo contribua para uma melhor compreensão do fenómeno social dos acidentes de trânsito, cujas consequências afectam não só o motorista envolvido, mas, quando resultantes de violações das regras de trânsito, questionam as regras sociais e comprometem a vida em sociedade e as interações entre os diferentes elementos que a compõem. Com base nisso, apresentamos as seguintes hipóteses:

1) Os motoristas com maior prevalência do traço procura de sensações apresentam maior predisposição para uma condução mais descuidada e arriscada, sendo essa propensão mais acentuada nos motoristas masculinos do que nos femininos.

2) O cometimento dos erros e das infrações que configuram a condução perigosa é mais frequente nos motoristas masculinos mais desinibidos e mais susceptíveis ao tédio, ao contrário dos lapsos que podem ser cometidos indistintamente por motoristas de ambos os sexos.

\section{Método}

\section{Participantes}

Participaram do estudo 140 motoristas portugueses residentes na área metropolitana de Lisboa, dos quais 70 homens e 70 mulheres, com idades entre os 25 e os 55 anos (média 44,99 \pm 8,28 anos para os homens e 41,19 $\pm 8,69$ anos para as mulheres). Todos tinham experiência de condução superior a dois anos (média 23,27 \pm 7,85 anos para os homens e 17,60 \pm 7,73 anos para as mulheres), e verificou-se que $90 \%$ dos homens e $84 \%$ das mulheres conduziam diariamente.

\section{Instrumentos}

O traço de personalidade procura de sensações foi avaliado com recurso à sensation seeking scale (SSS-V) de Zuckerman (1994), adaptada para português por Silva e Queirós (2012), composta por quatro subescalas que avaliam as seguintes dimensões: busca de emoção e aventura (Thrill and Adventure Seeking - TAS), busca de experiências (Experience Seeking - ES), desinibição (Disinhibition - DIS) e susceptibilidade ao tédio (Boredom Susceptibility - BS), que medem, respectivamente, a procura de experiências arriscadas e pouco usuais, a busca de novas sensações e experiências mentais, de atividades sociais intensas e de intolerância à repetição (Tabela 1). A escala é composta por 40 itens dicotómicos (presença ou ausência do traço), seguindo um modelo de resposta 
forçada em que cada subescala é composta por dez itens. O resultado de cada subescala pode variar entre 0 e 10, enquanto o resultado total pode variar de 0 a 40, calculando-se o valor total do traço de procura de sensações, com base no somatório dos valores obtidos com as quatro subescalas. Todas as subescalas apresentam valores de consistência interna aceitáveis, variando o alfa de Cronbach entre 0,66 e 0,76.

Tabela I. Médias, desvio padrão e teste das diferenças para as dimensões do driver behavior questionnaire (DBQ) e da SSS-V em função do sexo

\begin{tabular}{|c|c|c|c|c|c|c|c|}
\hline \multirow{2}{*}{\multicolumn{2}{|c|}{ Dimensões }} & \multicolumn{4}{|c|}{ Média (DP) } & \multirow[b]{2}{*}{ Mann-Whitney U } & \multirow[b]{2}{*}{$p$} \\
\hline & & \multicolumn{2}{|c|}{ Masculino } & \multicolumn{2}{|c|}{ Feminino } & & \\
\hline \multirow[t]{3}{*}{ DBQ } & Lapsos (0-8) & $\mathrm{I}, 25$ & $(, 55)$ & 1,23 & $(, 60)$ & 2352,0 & ,682 \\
\hline & Erros (0-8) & ,84 & $(, 42)$ & ,72 & $(, 47)$ & 1997,0 & ,058 \\
\hline & Infrações (0-8) & $\mathrm{I}, 30$ & $(, 72)$ & ,98 & $(, 57)$ & 1854,0 & ,013* \\
\hline \multirow[t]{5}{*}{ SSS-V } & TAS $(0-10)$ & 5,0 & $(2,68)$ & 4,00 & $(2,86)$ & 1942,0 & ,033* \\
\hline & ES $(0-10)$ & 4,73 & $(I, 83)$ & 5,43 & $(1,90)$ & 1919,5 & ,025* \\
\hline & DIS (0-I0) & 3,40 & $(1,50)$ & 3,03 & $(1,50)$ & 2134,5 & , I79 \\
\hline & BS $(0-10)$ & 2,33 & $(1,45)$ & 2,80 & $(1,65)$ & 2033,5 & ,076 \\
\hline & SStotal $(0-40)$ & 15,46 & $(4,30)$ & 15,26 & $(5,65)$ & 2431,5 & ,938 \\
\hline
\end{tabular}

* $p<0,050$.

TAS = busca de emoção e aventura; ES = busca de experiências; DIS = desinibição; BS = susceptibilidade ao tédio; SStotal $=$ prevalência geral do traço.

Fonte: Elaborada pelos autores.

A propensão para acidentes resultantes dos comportamentos de condução foi avaliada através do driver behavior questionnaire (DBQ) de Reason et al. (1990), adaptado para português por Correia (2008), tendo 24 itens organizados em três factores de oito itens cada:

- Lapsos que configuram falhas de atenção e de memória e que são menos passíveis de resultar em acidentes graves.

- Erros enquanto ações que se traduzem na falha dos resultados que se pretendiam alcançar e que colocam, de algum modo, a segurança do motorista em risco.

- Infrações de trânsito ou transgressões enquanto condutas deliberadamente contrárias às práticas consideradas necessárias para manter seguro um sistema potencialmente perigoso, estando fortemente associadas aos acidentes de trânsito.

Todas as subescalas apresentam valores de consistência interna aceitáveis, variando o alfa de Cronbach entre 0,61 e 0,73. 


\section{Procedimentos}

Toda a investigação respeitou os procedimentos éticos e de confidencialidade dos dados, que foram previamente definidos e autorizados para o desenvolvimento deste projeto. A recolha dos dados foi feita numa base individual, com recurso a questionários de autopreenchimento que foram distribuídos e preenchidos presencialmente com o apoio de colaboradores que se empenharam na identificação de participantes voluntários através do método "bola de neve". Aos participantes foi dado um termo de consentimento informado onde era explicada a natureza do projeto de investigação e garantido o anonimato e a confidencialidade dos dados. Os questionários tinham instruções precisas sobre o seu preenchimento.

Os dados recolhidos foram processados com recurso ao programa de estatística SPSS 20. Foi efectuada uma análise descritiva, bem como correlações parciais de Pearson. Para melhor compreender a predição da interação dos traços de personalidade com os vários tipos de comportamento de condução, foi feita uma análise de regressão. Os testes de normalidade mostram que, de acordo com o teste Kolmorogov-Smirnov, a falta de normalidade da distribuição da maioria das variáveis recomenda a adopção de testes não paramétricos. Contudo, por termos mais de 30 participantes na amostra (140 motoristas) e de acordo com o teorema do limite central da teoria da probabilidade, à medida que a dimensão da amostra aumenta, a distribuição da média aproxima-se da distribuição normal (Maroco, 2010), sendo permitido o uso de testes paramétricos.

\section{Resultados}

Quanto aos três comportamentos de risco na condução, podemos constatar (Tabela 1) que, relativamente às mulheres, os motoristas masculinos cometem mais lapsos de condução, erros e infrações, embora essas diferenças apenas tenham relevância significativa nas infrações. Por sua vez e no que respeita às dimensões da personalidade relacionadas com a procura de sensações (Tabela 1), os motoristas masculinos apresentam valores superiores para as dimensões (TAS), (DIS) e total da escala, e valores inferiores ao das mulheres nas dimensões (ES) e (BS), embora com diferenças significativas apenas nas dimensões (TAS) e (ES).

Relativamente às correlações parciais entre as várias dimensões do traço de personalidade procura de sensações e os comportamento de condução (Tabela 2), verificamos que são mais fortes no sexo feminino do que no masculino. Com efeito, nos motoristas femininos, registamos uma correlação significativa entre a (ES) e as infrações, e correlações moderadas entre a (DIS) e os lapsos e as infrações, bem como entre o total do traço e as infrações. Por sua vez, nos motoristas masculinos, apenas registamos uma correlação significativa mas de sentido inverso entre o total do traço e o cometimento de lapsos. 
Tabela 2. Correlações parciais de Pearson entre personalidade e comportamento de condução, em função do sexo

\begin{tabular}{|c|c|c|c|c|c|c|c|c|c|}
\hline Feminino & Dimensões & 1 & 2 & 3 & 4 & 5 & 6 & 7 & 8 \\
\hline \multirow[t]{3}{*}{ DBQ } & I. Lapsos & - & ,619** & ,442*** &,- 209 &,- 140 &,- 170 &,- 043 &,$- 263 *$ \\
\hline & 2. Erros &, $575^{*} *$ & - & ,509*** &,- 044 &,- 205 &,- 065 & , 106 &,- 101 \\
\hline & 3. Infrações & ,49I*** &, $552 * *$ & - & ,053 &,- 199 &, 105 & ,059 & ,005 \\
\hline \multirow[t]{5}{*}{ SSS-V } & 4. TAS &,- 010 &,- 182 & ,221 & - &, $243^{*}$ & , 155 &,- 134 & ,735** \\
\hline & 5. ES & , 173 &, 049 & ,268* & ,45I米 & - & ,279* &,- 135 & ,627*** \\
\hline & 6. DIS &, $375 * *$ &, 221 & ,455*** &, $307^{* *}$ &, $513 * *$ & - & ,059 & ,583** \\
\hline & 7. BS & ,054 &,- 032 &, 131 &, 145 &, 190 & ,430** & - &, 217 \\
\hline & 8. SStotal & , 169 &,- 027 & ,36I*** & ,782*** & ,757*** & ,719** &, $543 * *$ & - \\
\hline
\end{tabular}

* $p<0,050$. *** $p<0,01$.

Nota: As correlações femininas e masculinas são apresentadas respectivamente abaixo e acima da diagonal.

Fonte: Elaborada pelos autores.

A análise da regressão múltipla Stepwise (Tabela 3) permite-nos verificar que, relativamente aos motoristas masculinos, o total do traço procura de sensações apenas explica de forma negativa $7 \%$ dos lapsos, enquanto, nos motoristas femininos, a desinibição explica $14 \%$ dos lapsos e $21 \%$ das infrações.

Tabela 3. Análise de regressão da personalidade como preditora do comportamento de condução

\begin{tabular}{|c|c|c|c|c|c|c|c|c|c|}
\hline \multirow[b]{3}{*}{ Sexo } & \multirow[b]{3}{*}{ Personalidade } & \multicolumn{8}{|c|}{ Comportamento do condutor } \\
\hline & & \multicolumn{4}{|c|}{ DBQ-Lapsos } & \multicolumn{4}{|c|}{ DBQ-Infrações } \\
\hline & & $\mathrm{R}^{2}$ & $\beta$ & $\mathrm{F}$ & $\mathrm{t}$ & $\mathrm{R}^{2}$ & $\beta$ & $\mathrm{F}$ & $\mathrm{t}$ \\
\hline Homens & SS total & 069 &,- 263 & $5,064 *$ & $-2,250 *$ & - & - & - & - \\
\hline Mulheres & DIS &, 140 & ,375 & II,096* & $3,33 I^{*}$ & ,207 & ,455 & $17,765^{*}$ & $4,215^{*}$ \\
\hline
\end{tabular}

* $p<0,050$.

Fonte: Elaborada pelos autores.

\section{Discussão}

O elevado número de acidentes rodoviários e os avultados prejuízos de natureza socioeconómica que estes provocam em cada país têm levado os investigadores a tentar conhecer o perfil dos comportamentos de risco que ajudem a prevenir os acidentes. Os resultados obtidos junto de uma amostra composta por indivíduos com razoável experiência de condução em ambiente urbano, onde em regra se verificam as situações 
mais problemáticas e mais stressantes em matéria de trânsito, revelaram que os motoristas masculinos relatam o cometimento de um maior número de infrações do que os motoristas femininos, não sendo identificáveis diferenças significativas ao nível do cometimento de lapsos e erros de condução.

O sexo tem sido relacionado com o tipo de condução e com o comportamento da condução, mesmo considerando a idade e a experiência de condução (Reason et al., 1990). Os homens reportam mais acidentes, mais multas de trânsito e mais infrações do que as mulheres (Lonczak et al., 2007). Além disso, os homens apresentam determinadas características da personalidade que os levam a subestimar o perigo e a assumir um maior número de riscos na condução (Constantinou et al., 2011).

Neste estudo, considerámos, na hipótese 1, que os motoristas com maior prevalência da procura de sensações teriam uma maior predisposição para a condução descuidada e arriscada, sendo essa tendência maior nos homens do que nas mulheres, à semelhança do que se tem verificado em estudos anteriores. Contudo, os resultados sugerem poucas diferenças significativas entre os sexos, talvez pelo reduzido tamanho da amostra e pela idade média dos motoristas que é relativamente elevada, comparativamente à de outros estudos que trabalharam com amostras de motoristas mais jovens, o que, segundo a literatura, apresenta uma maior predisposição para o cometimento de mais infrações e para a prática de condução mais arriscada (Cooper et al., 1994).

No que se refere à hipótese 2 , considerámos a possibilidade de os homens mais desinibidos e mais susceptíveis ao tédio poderem cometer mais erros e mais infrações, e de os lapsos poderem ser cometidos indiscriminadamente pelos motoristas de ambos os sexos. Verificamos que, nestas dimensões da personalidade, os motoristas masculinos, ao contrário dos motoristas femininos, não apresentam diferenças significativas quanto ao cometimento de erros e infrações, e que os lapsos de condução são cometidos indistintamente pelos motoristas de ambos os sexos, havendo uma correlação significativa entre os três comportamentos de condução.

Verificamos que a dimensão da personalidade desinibição (DIS) é aquela que meIhor prediz a ocorrência de lapsos e infrações, sobretudo nos motoristas femininos, ao contrário do total do traço procura de sensações que apenas revela uma predição inversa ou negativa de $7 \%$ nos lapsos dos motoristas masculinos. Tal associação negativa permite-nos inferir que, ao invés dos motoristas femininos, os homens com maior escore no total do traço procura de sensações são menos propensos ao cometimento de lapsos de condução e por conseguinte poderão adoptar um estilo de condução mais consciente, embora não necessariamente menos arriscado. À semelhança do que tem sido apurado nalguns estudos internacionais, a desinibição é uma das dimensões da personalidade que potenciam o cometimento de infrações e um estilo de condução mais arriscada (Constantinou et al., 2011).

Algumas investigações têm evitado o tratamento da personalidade por se considerar que é uma variável estável e bastante resistente à mudança e de difícil avaliação. Os nossos resultados apontam para o facto de a personalidade poder ter influência 
diferencial em cada sexo nos comportamentos de condução, nomeadamente no cometimento de lapsos, erros e infrações que configuram situações de condução perigosa. À semelhança de outros estudos, podemos verificar que as características pessoais afectam a motivação e o estilo individual de condução, havendo traços de personalidade que podem constituir factores de risco e propensão para os acidentes. A desinibição, por exemplo, quando surge nas mulheres, tende a potenciar a prática de lapsos e de infrações, constituindo-se como um factor de risco adicional que deve ser tido em conta na caracterização do seu comportamento de condução.

O fracasso de algumas campanhas de prevenção de acidentes, particularmente nos grupos dos motoristas de alto risco, pode estar relacionado com a forma como cada sexo percepciona os riscos na condução e as atitudes que adopta para os minimizar. Apesar de em futuros estudos ser necessário considerar a utilização de amostras de maior dimensão, por forma a agrupar os participantes por idades e experiência de condução, e, por essa via, melhor fazer realçar e explorar as tendências comportamentais relacionadas com cada sexo, os resultados obtidos podem ser considerados úteis para a formação de futuros motoristas, sendo importante dispor de treinos direcionados para as características individuais, em detrimento de tipologias de formação iguais para todos os candidatos. Alguns autores (Sampaio \& Nakano, 2011; Silva \& Alchieri, 2007) alertam que esta obrigatoriedade pode não contribuir para uma maior segurança no trânsito, fruto das incertezas que ainda subsistem sobre o perfil psicológico dos motoristas, a padronização dos instrumentos psicométricos e a respetiva validade preditiva no contexto do trânsito. Contudo, conhecer as características da personalidade e as diferenças individuais, como o sexo, que possam ser trabalhadas de forma a prevenir a ocorrência de violações das regras de trânsito que frequentemente estão na origem de acidentes, poderá contribuir para uma vida em sociedade que implique respeito pelas regras e uma saudável convivência entre cidadãos que utilizam as vias de circulação. Tal como referem Oliveira e Pinheiro (2007), compreender os acidentes através dos seus múltiplos determinantes é fundamental para que cada elemento deste fenómeno social contribua para um trânsito mais seguro e sem custos de tantas vidas humanas.

\section{Referências}

Bartholomeu, D. (2008). Traços de personalidade e comportamentos de risco no trânsito: um estudo correlacional. Psicologia Argumento, 26(54), 193-206.

Cestac, J., Paran, F., \& Delhomme, P. (2011). Young drivers' sensation seeking, subjective norms, and perceived behavioral control and their roles in predicting speeding intention: How risk-taking motivations evolve with gender and driving experience. Safety Science, 49, 424-432. Recuperado de http://10.0.3.248/j.ssci. 2010.10.007. 
Constantinou, E., Panayiotou, G., Konstantinou, N., Loutsiou-Ladd, A., \& Kapardis, A. (2011). Risky and aggressive driving in young adults: personality matters. Accident Analysis and Prevention, 43, 1323-1331. Recuperado de http://10.0.3.248/ j.aap.2011.02.002.

Cooper, P. J., Pinili, M., \& Chen, W. (1994). An examination of the crash involvement rates of novice drivers aged 16 to 55. Accident Analysis and Prevention, 7, 89-104.

Correia, J. P. (2008). A atitude e a motivação do bem conduzir. Porto: Livpsic-Legis.

Fernandes, R., Hatfield, J., \& Job, R. F. S. (2010). A systematic investigation of the differential predictors for speeding, drink-driving, driving while fatigued, and not wearing a seat belt, among young drivers. Transportation Research Part $F$, 13, 179-196. Recuperado de http://10.0.3.248/j.trf.2010.04.007.

Jonah, B. A. (1997). Sensation seeking and risky driving: a review and synthesis of the literature. Accident Analysis and Prevention, 29, 651-665.Recuperado de http:// 10.0.3.248/S0001-4575(97)00017-1.

Jonah, B. A., Thiessen, R., \& Au-Yeung, E. (2001). Sensation seeking, risky driving and behavioral adaptation. Accident Analysis and Prevention, 33, 679-684. Recuperado de http://10.0.3.248/S0001-4575(00)00085-3.

Jovanović, D., Stanojević, P., \& Stanojević, D. (2011). Motives for, and attitudes about, driving-related anger and aggressive driving. Social Behavior and Personality, 39(6), 755-764.

Lonczak, H. S., Neighbors, C., \& Donovan, D. M. (2007). Predicting risky and angry driving as a function of gender. Accident Analysis and Prevention, 39, 536-545. Recuperado de: http://10.0.3.248/j.aap.2006.09.010.

Maroco, J. (2010). Análise estatística com o PASW. Pêro Pinheiro: Report Number.

National Highway Traffic Safety Administration, Washington, U.S. Department of Transportation. (2013, April). Traffic safety facts: 2011 Data. Recuperado em 18 maio, 2013, de http://www-nrd.nhtsa.dot.gov/Pubs/811744.pdf.

NSW (2011). Road and Maritime Services, Government of Australia. Recuperado em 15 março, 2013, de http://www.rta.nsw.gov.au/licensing/tests/driverqualificationtest/ sensationseekingscale/index.html/.

Oliveira, A. C. F., \& Pinheiro, J. Q. (2007). Indicadores psicossociais relacionados a acidentes de trânsito envolvendo motoristas de ônibus. Psicologia em Estudo, 12(1), 171-178. Recuperado de http://10.0.6.54/S1413-73722007000100020.

Özkan, T., \& Lajunen, T. (2006). What causes the differences in driving between young men and women? The effects of gender roles and sex on young drivers' driving behaviour and self-assessment of skills. Transportation Research Part F, 9, 269-277. 
Reason, J., Manstead, A., Stradling, S., Baxter, J., \& Campbell, K. (1990). Errors and violations on the road: a real distinction? Ergonomics, 33, 1315-1332. Recuperado de http://10.0.4.56/00140139008925335.

Sampaio, M. H. L., \& Nakano, T. C. (2011). Avaliação psicológica no contexto do trânsito: revisão de pesquisas brasileiras. Psicologia: Teoria e Prática, 13(1), 15-33.

Santos, P. L., Boff, R. M., \& Konflanz, S. S. (2012). Relevância da avaliação obrigatória de traços de personalidade em motoristas. Psicologia: Teoria e Prática, 14(3), 101-110.

Silva, A. L., \& Queirós, C. (2012). Sensation seeking and burnout police officers. In S. P. Gonçalves \& J. Neves (Eds.). Ocupational health psychology: from burnout to well-being (pp.93-125). Rosemead: Scientific \& Academic Publishing.

Silva, F. H. V. C., \& Alchieri, J. C. (2007). Avaliação psicológica da personalidade de condutores: uma revisão de literatura. PsicoUSF, 12(2), 189-196. Recuperado de http://10.0.6.54/S1413-82712007000200007.

Zuckerman, M. (1994). Behavioural expressions and biosocial bases of sensation seeking. Cambridge: University of Cambridge Press.

Submissão: 22.5 .2013

Aceitação: 15.10.2013 